\title{
Perturbative Calculation of Multi-Loop Feynman Diagrams. New Type of Expansions for Critical Exponents
}

\author{
Hagen Kleinert* \\ Institut für Theoretische Physik, \\ Freie Universität Berlin, Arnimallee 14, 14195 Berlin, Germany
}

We show that the calculation of $L$-loop Feynman integrals in $D$ dimensions can be reduced to a series of matrix multiplications in $D \times L$ dimensions. This leads to a new type of expansions for the critical exponents in three dimensions in which all coefficients are calculable exactly.

1. Recently, an efficient method has been developed for constructing all Feynman diagrams of a quantum field theory recursively to arbitrary order in perturbation theory [i]. The construction has also been automatized by computer algebra [1], so that one can now easily list all diagrams to any desired order, limited only by computer time.

The only open problem is an efficient evaluation of the associated Feynman integrals. The highest loop number so far has been treated in $\phi^{4}$-theory where renormalization constants of field normalization and mass are now known numerically up to seven loops [2].

For a reliable calculation of all critical exponents with an accuracy which has become recently available in satellite experiments on superfluid helium [3] it is desirable to know the perturbation expansions to even higher order [1.6. 6 . In this note we would like to propose a perturbative evaluation of many-loop Feynman integrals which reduces the integrations over $D \times L$ loop momenta to a series of multiplications of $D \times L$-dimensional matrices, thus reducing greatly the number of numerical operations.

2. The method is most easily introduced by treating an exactly solvable class of integrals

$$
I_{a}^{D}=\int \frac{d^{D} p}{(2 \pi)^{D}} \frac{1}{\left(1+\mathbf{p}^{2}\right)^{a}}
$$

whose integration via Schwinger's proper-time technique yields

$$
I_{a}^{D}=\frac{1}{(4 \pi)^{D / 2}} \frac{\Gamma(a-D / 2)}{\Gamma(a)} .
$$

Our perturbative evaluation starts out by introducing into (ii) a smallness parameter $\epsilon$, and considering the functions of $\epsilon$

\footnotetext{
*Email: kleinert@physik.fu-berlin.de URL: http://www.physik.fu-berlin.de/ ${ }^{\sim}$ kleinert
}

$$
I_{a}^{D}(\epsilon)=\int \frac{d^{D} p}{(2 \pi)^{D}} \frac{1}{\left(1+\epsilon \mathbf{p}^{2}\right)^{a / \epsilon}} .
$$

For this function, we can easily find a perturbation expansion in powers of $\epsilon$, by rewriting it as follows:

$$
I_{a}^{D}(\epsilon)=\frac{1}{(4 \pi a)^{D / 2}} \int \frac{d^{D} p}{(\pi / a)^{D / 2}} e^{-a \mathbf{p}^{2}-S_{\epsilon}(\mathbf{p})},
$$

with

$$
\begin{aligned}
S_{\epsilon}(\mathbf{p}) & \equiv \frac{a}{\epsilon} \log \left(1+\epsilon \mathbf{p}^{2}\right)-a \mathbf{p}^{2} \\
& =-a\left[\frac{\epsilon}{2}\left(\mathbf{p}^{2}\right)^{2}-\frac{\epsilon^{2}}{3}\left(\mathbf{p}^{2}\right)^{3}+\frac{\epsilon^{3}}{4}\left(\mathbf{p}^{2}\right)^{4}+\ldots\right] .
\end{aligned}
$$

Considering $a \mathbf{p}^{2}$ as a free "action", and $S_{\epsilon}(\mathbf{p})$ as an "interaction", we introduce the Gaussian expectation values

$$
\langle f(\mathbf{p})\rangle \equiv \int \frac{d^{D} p}{(\pi / a)^{D / 2}} e^{-a \mathbf{p}^{2}} f(\mathbf{p}),
$$

and rewrite the integral (44) as a harmonic expectation

$$
I_{a}^{D}(\epsilon)=\frac{1}{(4 \pi a)^{D / 2}}\left\langle e^{-S_{\epsilon}(\mathbf{p})}\right\rangle .
$$

The exponential $e^{-S_{\epsilon}(\mathbf{p})}$ is now expanded in a Taylor series, and the expectation values $\left\langle S_{\epsilon}(\mathbf{p}) \cdots S_{\epsilon}(\mathbf{p})\right\rangle$ are calculated via a Wick expansion using the basic correlation function

$$
\left\langle p_{i_{1}} p_{i_{2}}\right\rangle=G_{i_{1} i_{2}}=\frac{1}{2 a} \delta_{i_{1} i_{2}}, \quad i=1, \ldots, D .
$$

The nonzero expectation values of higher products

$$
\left\langle p_{i_{1}} \cdots p_{i_{2 n}}\right\rangle=G_{i_{1} \ldots i_{2 n}}
$$

are calculated from the recursion relation

$$
\begin{aligned}
& G_{i_{1} \ldots i_{2 n}}=G_{i_{1} i_{2}} G_{i_{3} i_{4} \ldots i_{2 n-1} i_{2 n}}+G_{i_{1} i_{3}} G_{i_{2} i_{4} \ldots i_{2 n}} \\
& +\ldots+G_{i_{1} i_{2 n-1}} G_{i_{2} i_{3} \ldots i_{2 n}}+G_{i_{1} i_{2 n}} G_{i_{2} i_{3} \ldots i_{2 n-1}}
\end{aligned}
$$

For example, we find

$$
\begin{aligned}
& I_{1}^{1}(\epsilon)=\frac{1}{(4 \pi)^{1 / 2}}\left(1+\frac{3 \epsilon}{8}+\frac{25 \epsilon^{2}}{128}+\frac{105 \epsilon^{3}}{1024}+\frac{1659 \epsilon^{4}}{32768}+\ldots\right), \\
& I_{2}^{3}(\epsilon)=\frac{1}{(8 \pi)^{3 / 2}}\left(1+\frac{15 \epsilon}{16}+\frac{385 \epsilon^{2}}{512}+\frac{4725 \epsilon^{3}}{8192}+\frac{228459 \epsilon^{4}}{524288}+\ldots\right) .
\end{aligned}
$$

The corresponding cumulant expansions are 


$$
\begin{aligned}
& I_{1}^{1}(\epsilon)=\frac{1}{(4 \pi)^{1 / 2}} e^{\frac{3 \epsilon}{8} \epsilon+\frac{1}{8} \epsilon^{2}+\frac{3}{64} \epsilon^{3}+\frac{1}{64} \epsilon^{4}+\ldots}, \\
& I_{2}^{3}(\epsilon)=\frac{1}{(8 \pi)^{3 / 2}} e^{\frac{15}{16} \epsilon+\frac{5}{16} \epsilon^{2}+\frac{75}{512} \epsilon^{3}+\frac{41}{512} \epsilon^{4}+\ldots} .
\end{aligned}
$$

Evaluating the exponents at $\epsilon=1$ successively as partial sums of orders $1,2,3, \ldots, 15$ [not all terms are written down in (12) and (13), for lack of space], the relative deviations from the respective exact values $1 / 2$ and $1 / 8 \pi$ [which follow from Eq. (2)] are shown in Table [ [columns marked with (ps)]. The deviation sequences tend slowly toward zero, and grow again for larger order as a consequence of the asymptotic nature of the expansions.

The partial sums yield integrals whose first ten terms tend exponentially fast toward the exact results, as shown in Figs. 1 .
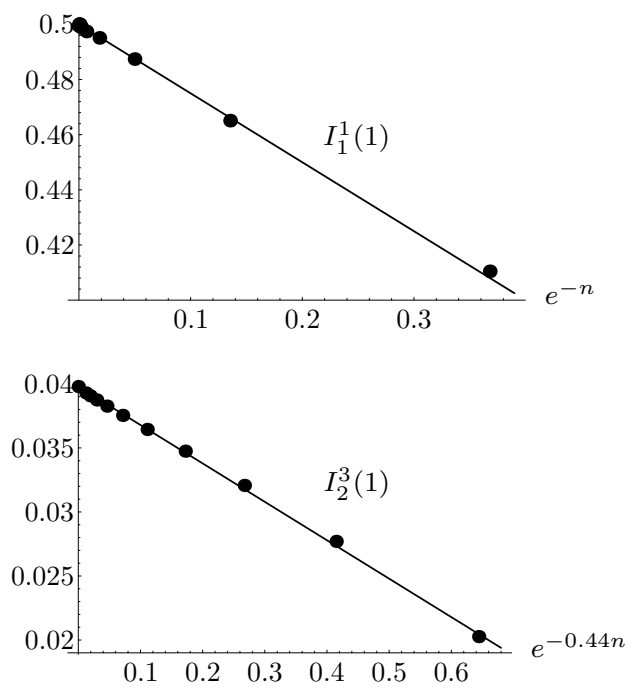

FIG. 1. Exponentially fast convergence of the first ten approximations, obtained from the successive partial sums of the exponents in (12) and (13), against their respective exact values $1 / 2$ and $1 / 8 \pi \approx 0.397884 \ldots$. The number $n$ denotes the order of the approximations.

The convergence is accelerated by approximating the successive exponents à la Padé, leading rapidly to accurate results, as we can see in the right-hand columns of Table 1 .

3. As a nontrivial example consider now the Feynman integral

$$
\begin{aligned}
I^{D}= & \int \frac{d^{D} p_{1}}{(2 \pi)^{D}} \int \frac{d^{D} p_{2}}{(2 \pi)^{D}} \int \frac{d^{D} p_{3}}{(2 \pi)^{D}} \\
& \times \frac{1}{1+\mathbf{p}_{1}^{2}} \frac{1}{1+\mathbf{p}_{2}^{2}} \frac{1}{1+\mathbf{p}_{3}^{2}} \frac{1}{1+\left(\mathbf{p}_{1}+\mathbf{p}_{2}+\mathbf{p}_{3}\right)^{2}} .
\end{aligned}
$$

We introduce again the smallness parameter $\epsilon$ as in (3), and rewrite the integral (14) as

$$
I^{D}(\epsilon)=\int \frac{d^{D} p_{1}}{(2 \pi)^{D}} \int \frac{d^{D} p_{2}}{(2 \pi)^{D}} \int \frac{d^{D} p_{3}}{(2 \pi)^{D}}
$$

$$
\times e^{-\left[\mathbf{p}_{1}^{2}+\mathbf{p}_{2}^{2}+\mathbf{p}_{3}^{2}+\left(\mathbf{p}_{1}+\mathbf{p}_{2}+\mathbf{p}_{3}\right)^{2}\right]-S_{\epsilon}\left(\mathbf{p}_{1}, \mathbf{p}_{2}, \mathbf{p}_{3}\right)},
$$

with a free "action" $\mathbf{p}_{1}^{2}+\mathbf{p}_{2}^{2}+\mathbf{p}_{3}^{2}+\left(\mathbf{p}_{1}+\mathbf{p}_{2}+\mathbf{p}_{3}\right)^{2}$, and an "interaction"

$$
\begin{aligned}
& S_{\epsilon}\left(\mathbf{p}_{1}, \mathbf{p}_{2}, \mathbf{p}_{3}\right)=\sum_{a=1}^{3}\left[\frac{1}{\epsilon} \log \left(1+\epsilon \mathbf{p}_{a}^{2}\right)-\mathbf{p}_{a}^{2}\right] \\
&+\frac{1}{\epsilon} \log \left[1+\epsilon\left(\sum_{a=1}^{3} \mathbf{p}_{a}\right)^{2}\right]-\left(\sum_{a=1}^{3} \mathbf{p}_{a}\right)^{2} .
\end{aligned}
$$

The free action is now rewritten as a quadratic form $\frac{1}{2} p_{a i} K_{a i, b j} p_{b j}$, with the matrix

$$
K_{a i, b j}=k_{a b} \delta_{i j}, \quad k_{a b}=2\left(\begin{array}{lll}
2 & 1 & 1 \\
1 & 2 & 1 \\
1 & 1 & 2
\end{array}\right), \quad a=1,2,3 .
$$

where $\delta_{i j}$ is the $D$-dimensional Kronecker symbol. We now define harmonic expectation values

$$
\begin{aligned}
& \left\langle f\left(\mathbf{p}_{1}, \mathbf{p}_{2}, \mathbf{p}_{3}\right)\right\rangle \equiv(2 \pi)^{3 D / 2}(\operatorname{det} K)^{1 / 2} \\
& \times \int \frac{d^{D} p_{1}}{(2 \pi)^{D}} \int \frac{d^{D} p_{2}}{(2 \pi)^{D}} \int \frac{d^{D} p_{3}}{(2 \pi)^{D}} f\left(\mathbf{p}_{1}, \mathbf{p}_{2}, \mathbf{p}_{3}\right) e^{-\frac{1}{2} p_{a i} K_{a i, b j} p_{b j}}
\end{aligned}
$$

where

$$
\operatorname{det} K=\operatorname{det} k^{D}=32^{D} .
$$

The basic correlation functions are

$$
\begin{aligned}
\left\langle p_{a i} p_{b j}\right\rangle & =K_{a i, b j}^{-1}=G_{a i, b j}=g_{a b} \delta_{i j}, \\
g_{a b} & =k_{a b}^{-1}=\frac{1}{8}\left(\begin{array}{rrr}
3 & -1 & -1 \\
-1 & 3 & -1 \\
-1 & -1 & 3
\end{array}\right) .
\end{aligned}
$$

In terms of (17), the integral (15) can now be considered as a harmonic expectation value

$$
I^{D}(\epsilon)=(2 \pi)^{-3 D / 2}(\operatorname{det} K)^{-1 / 2}\left\langle e^{-S_{\epsilon}\left(\mathbf{p}_{1}, \mathbf{p}_{2}, \mathbf{p}_{3}\right)}\right\rangle,
$$

which is evaluated perturbatively by expanding the exponential into a power series, and forming all Wick contractions. The results are for $D=1$ and 2 :

$$
\begin{aligned}
& I^{1}(\epsilon)=\frac{1}{(2 \pi)^{3 / 2}} \frac{1}{\sqrt{32}} e^{\frac{27}{32} \epsilon+\frac{9}{64} \epsilon^{2}+\frac{24}{4096} \epsilon^{3}+\frac{249}{8192} \epsilon^{4}+\ldots,} \\
& I^{2}(\epsilon)=\frac{1}{(2 \pi)^{3}} \frac{1}{\sqrt{32^{2}}} e^{\frac{9}{4} \epsilon+\frac{21}{32} \epsilon^{2}+\frac{13}{64} \epsilon^{3}+\frac{177}{1024} \epsilon^{4}+\ldots},
\end{aligned}
$$

and the successive evaluation of the exponent at $\epsilon=1$ yields the approximations

$$
\begin{array}{llll}
0.026097, & 0.030038, & 0.030236, & 0.031169 \\
0.001195, & 0.002304, & 0.002823, & 0.003356
\end{array}
$$

which converge against the precise values of the Feynman integral $1 / 32=0.03125$ and $0.00424027 \ldots$, respectively. 
These tend exponentially fast against the exact value, as seen in Fig. 2.
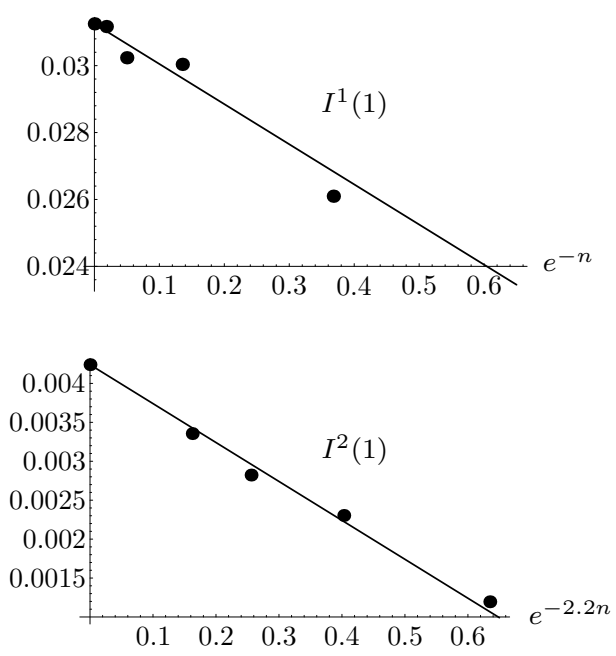

FIG. 2. Exponentially fast convergence of the approximation sequences (20) and (21) against their respective exact values $1 / 32$ and $0.00424027 \ldots$. The number $n$ denotes the order of the approximations.

The same Padé approximants as before yield the numbers

$$
\begin{array}{lll}
0.030894, & 0.030171, & 0.03064, \\
0.003019, & 0.003091, & 0.00309,
\end{array}
$$

which, surprisingly, show no improvement with respect to the sequences (20), (21).

4. So far we have calculated only vacuum diagrams. There is no problem in extending the method to diagrams with external legs. The first nontrivial Feynman integral in the self-energy of a $\phi^{4}$-theory, for example, is

$$
\begin{aligned}
I^{D}(\mathbf{q})= & \int \frac{d^{D} p_{1}}{(2 \pi)^{D}} \int \frac{d^{D} p_{2}}{(2 \pi)^{D}} \\
& \times \frac{1}{1+\left(\mathbf{p}_{1}+\mathbf{q}\right)^{2}} \frac{1}{1+\mathbf{p}_{2}^{2}} \frac{1}{1+\left(\mathbf{p}_{1}+\mathbf{p}_{2}\right)^{2}} .
\end{aligned}
$$

which we rewrite as

$$
\begin{aligned}
I^{D}(\mathbf{q} ; \epsilon)= & e^{-\mathbf{q}^{2}} \int \frac{d^{D} p_{1}}{(2 \pi)^{D}} \int \frac{d^{D} p_{2}}{(2 \pi)^{D}} \\
& \times e^{-\left[\mathbf{p}_{1}^{2}+\mathbf{p}_{2}^{2}+\left(\mathbf{p}_{1}+\mathbf{p}_{2}\right)^{2}\right]-S_{\epsilon}\left(\mathbf{p}_{1}, \mathbf{p}_{2}, \mathbf{q}\right)-2 \mathbf{p}_{1} \mathbf{q}},
\end{aligned}
$$

with a free "action" $\mathbf{p}_{1}^{2}+\mathbf{p}_{2}^{2}+\left(\mathbf{p}_{1}+\mathbf{p}_{2}\right)^{2}$, a $\mathbf{q}$-dependent "interaction"

$$
\begin{aligned}
& S_{\epsilon}\left(\mathbf{p}_{1}, \mathbf{p}_{2}, \mathbf{q}\right)=\sum_{a=1}^{3}\left[\frac{1}{\epsilon} \log \left(1+\epsilon \mathbf{p}_{a}^{2}\right)-\mathbf{p}_{a}^{2}\right] \\
&+\frac{1}{\epsilon} \log \left[1+\epsilon\left(\sum_{a=1}^{3} \mathbf{p}_{a}\right)^{2}\right]-\left(\sum_{a=1}^{3} \mathbf{p}_{a}\right)^{2},
\end{aligned}
$$

where we have set $\mathbf{p}_{3} \equiv \mathbf{p}_{1}+\mathbf{p}_{2}$. After removing the extra source term $2 \mathbf{p}_{1} \mathbf{q}$ by a shift of the integration variable $\mathbf{p}_{1} \rightarrow \mathbf{p}_{1}-\mathbf{q}$, which produces an exponential factor $e^{2 k_{11} q^{2}}$, the integral can be treated as before, yielding an expansion in powers of $\epsilon$ with $\mathbf{q}^{2}$-dependent coefficients.

5. The above treatment of Feynman diagrams offers the interesting possibility of deriving a completely new type of power series expansions for the critical exponents of $\phi^{4}$-theories in three dimensions, in which expansion coefficients are calculable exactly, in contrast to the known expansions of Ref. [2]. For this we observe that our power series for the Feynman integrals can be reexpanded in powers of $g$ by inserting $\epsilon \equiv g /(m+g)$. Then $I_{1}^{1}(\epsilon)$ and $I_{2}^{3}(\epsilon)$ become

$$
\begin{aligned}
& I_{1}^{1}(\bar{g})=\frac{1}{(4 \pi)^{1 / 2}} \exp \left(\frac{3 \bar{g}}{8}-\frac{\bar{g}^{2}}{4}+\frac{11 \bar{g}^{3}}{64}-\frac{\bar{g}^{4}}{8}+\ldots\right), \\
& I_{2}^{3}(\bar{g})=\frac{1}{(4 \pi)^{3 / 2}} \exp \left(\frac{15 \bar{g}}{16}-\frac{5 \bar{g}^{2}}{8}+\frac{235 \bar{g}^{3}}{512}-\frac{23 \bar{g}^{3}}{64}+\ldots\right),
\end{aligned}
$$

where $\bar{g} \equiv g / m$. The coefficients have now alternating signs. These expansions must be evaluated in the strongcoupling limit $\bar{g} \rightarrow \infty$. This is again possible with the help of diagonal Padé approximations, and the results are precisely the same as those in Table 1 . Note that the choice of the parameter $m$ is irrelevant in this limit.

Now we recall that the critical behavior of $\phi^{4}$-theory can also be derived from power series expansions of the renormalization constants in the strong-coupling limit $g_{B} / m_{B} \rightarrow \infty$, where $g_{B}$ and $m_{B}$ are the bare coupling strength and mass of the theory. Let us identify the above expansion parameter $\bar{g}$ with $c g_{B} / m_{B}$, where $c$ is an arbitrary parameter which may be chosen at the end to optimize the speed of convergence. Then we insert the expansions of the Feynman integrals in powers of $g_{B} / m_{B}$ into the Feynman diagrams of the perturbation expansions of the renormalization constants of the $\phi^{4}$-theory. This leads to a new type of series in powers of $g_{B} / m_{B}$ whose coefficients are determined exactly. By going to the strong-coupling limit $g_{B} / m_{B} \rightarrow \infty$ as described in Ref. [5.6], we can obtain the critical exponents in three dimensions in a completely new way.

From the connected vacuum diagrams we obtain an expansion of the form

$$
W=m_{B}^{3} Z=m_{B}^{3}\left(1+a_{1} \bar{g}_{B}+a_{2} \bar{g}_{B}^{2}+\ldots\right) .
$$

For a comparison with experiment one assumes the bare mass to go linearly to zero as the temperature $T$ approaches the critical temperature, i.e. $m_{B}^{2} \propto\left(T / T_{c}-1\right)$. The vacuum energy $W$ has then for small $m_{B}$ the scaling behavior $\left(T / T_{c}-1\right)^{2-\alpha} \propto m_{B}^{4-2 \alpha}$, where $\alpha=2-3 \nu$ is the critical exponent of the peak in the specific heat in three dimensions ( $\nu$ is the exponent of the correlation length). Since $\bar{g}_{B}$ grows for small $m_{B}$ like $1 / m_{B}$, the power series for $Z$ in (27) must behave like $Z \propto \bar{g}_{B}^{2 \alpha-1}$. The critical 
exponent $\alpha$ is therefore found from the strong-coupling limit of the power series

$$
\alpha\left(\bar{g}_{B}\right)=\frac{1}{2}\left(1+d \log Z / d \log \bar{g}_{B}\right),
$$

evaluated as explained in Ref. [5] [6].

Also the exponent $\omega$ governing the approach to scaling can be obtained from the series (28), since $\alpha\left(\bar{g}_{B}\right)$ approaches $\alpha$ for large $\bar{g}_{B}$ like $\alpha+$ const $/ \bar{g}_{B}^{\omega}$. Thus $\omega$ is found from the strong-coupling limit of the series $\omega\left(\bar{g}_{B}\right)=-1-d \log \alpha^{\prime}\left(\bar{g}_{B}\right) / d \log \bar{g}_{B} \alpha^{\prime}\left(\bar{g}_{B}\right)$ (see [7] for more details).

The third independent critical exponent may be derived from the perturbative corrections to the field normalization. For this we take the expansion for the selfenergy $\Sigma(\mathbf{q})$ and extract the coefficient of the $\mathbf{q}^{2}$ term. This is done most simply by differentiating all Feynman integrals of the two-point function using the rule (in $D$ dimensions)

$$
\frac{\partial}{\partial \mathbf{q}^{2}}=\frac{1}{2 D} \frac{\partial^{2}}{\partial q_{\mu} \partial q_{\mu}}
$$

and setting $\mathbf{q}=0$. The momentum $\mathbf{q}$ always flows through one or more lines of the diagram, allowing the differentiations to be performed as follows:

$$
\begin{aligned}
\frac{\partial^{2}}{\partial q_{\mu} \partial q_{\mu}} \frac{1}{(\mathbf{p}-\mathbf{q})^{2}+m^{2}}= & \frac{-2 D}{\left[(\mathbf{p}-\mathbf{q})^{2}+m^{2}\right]^{2}} \\
& +\frac{8(\mathbf{p}-\mathbf{q})^{2}}{\left[(\mathbf{p}-\mathbf{q})^{2}+m^{2}\right]^{3}}
\end{aligned}
$$

After setting $\mathbf{q}=0$ in the resulting Feynman integrals, the calculation of the field normalization proceeds using the above formulas for the vacuum diagrams.

Note that the $\epsilon$-dependent Feynman integrals are all finite for small $\epsilon$. However, as $\epsilon$ increasing towards unity, a pole is encountered at $\epsilon=2 / 3$. Thus, the expansion of a pole term $\propto 1 /(\epsilon-2 / 3)$ should be subtracted from the $\epsilon$ expansions of divergent integrals before one can evaluate perturbation series at $\epsilon=1$, i.e., before going to the strong-coupling limit of $\bar{g}_{B}$.

6. Summarizing we have shown that just as the generation of all Feynman diagrams [1], also their evaluation can now be automatized by computer algebra, with the additional advantage that all expansion coefficients emerge as exact numbers.

The actual calculation of diagrams with eight and and more loops will, of course, require a considerable amount of work. It remains to be seen whether the new perturbation expansions converge more rapidly against the exact results than the presently known purely numerical ones.
[1] H. Kleinert, A. Pelster, B. Kastening, M. Bachmann, Recursive Graphical Construction of Feynman Diagrams and Their Multiplicities in $\phi^{4}$ - and $\phi^{2} A$-Theory, Berlin Preprint 1999 (hep-th/9907168).

[2] D.B. Murray and B.G. Nickel, unpublished.

[3] J.A. Lipa, D.R. Swanson, J. Nissen, T.C.P. Chui and U.E. Israelson, Phys. Rev. Lett. 76, 944 (1996).

[4] H. Kleinert Theory and Satellite Experiment on Critical Exponent $\alpha$ of Specific Heat in Superfluid Helium, FUBerlin preprint 1999 (cond-mat/9906107).

[5] H. Kleinert, Phys. Rev. D 57, 2264 (1998) (E-Print aps1997jun25_001); addendum (cond-mat/9803268).

[6] H. Kleinert Seven Loop Critical Exponents from StrongCoupling $\phi^{4}$-Theory in Three Dimensions, FU-Berlin preprint 1998 (hep-th/9812197).

[7] H. Kleinert, Critical Exponents without beta-Function, FU-Berlin preprint 1999 (cond-mat/9906359)

TABLE I. Relative deviations of approximations $I_{1}^{1}(1)$ and $I_{2}^{3}(1)$ from their exact values, once from successive partial sums (ps), once from successive Padé approximations.

\begin{tabular}{r|rr||l|rl}
\hline \hline$n$ & $I_{1}^{1}(1) \mathrm{ps}$ & $I_{2}^{3}(1) \mathrm{ps}$ & {$[\mathrm{N}, \mathrm{M}]$} & $I_{1}^{1}(1)$ Padé & $I_{2}^{3}(1)$ Padé \\
\hline 1 & 0.17910 & 0.49063 & {$[0,1]$} & -0.391430 & -3.85230 \\
2 & 0.06980 & 0.30377 & {$[1,1]$} & 0.009816 & 0.18603 \\
3 & 0.02516 & 0.19394 & {$[1,2]$} & 0.002101 & 0.09851 \\
4 & 0.00981 & 0.12674 & {$[2,2]$} & 0.000935 & 0.02490 \\
5 & 0.00516 & 0.08416 & {$[3,3]$} & 0.001821 & 0.00309 \\
6 & 0.00256 & 0.05655 & {$[4,4]$} & 0.000637 & -0.00062 \\
7 & 0.00027 & 0.03836 & {$[5,5]$} & 0.000652 & -0.00062 \\
8 & -0.00021 & 0.02625 & {$[6,6]$} & 0.000325 & $4.834910^{-5}$ \\
9 & 0.00124 & 0.01810 & {$[7,7]$} & 0.000325 & $8.004810^{-6}$ \\
10 & 0.00114 & 0.01255 & {$[8,8]$} & 0.000192 & $3.387310^{-5}$ \\
11 & -0.00273 & 0.00875 & {$[9,9]$} & 0.000194 & $1.618110^{-5}$ \\
12 & -0.00275 & 0.00613 & {$[10,10]$} & 0.000127 & $1.869410^{-5}$ \\
13 & 0.01001 & 0.00432 & {$[11,11]$} & 0.000130 & $1.096010^{-5}$ \\
14 & 0.01000 & 0.00305 & {$[12,12]$} & 0.000090 & $1.114610^{-5}$ \\
15 & -0.05026 & 0.00216 & {$[13,13]$} & 0.000095 & $7.363510^{-6}$ \\
\hline \multicolumn{5}{|c}{} \\
\hline \hline
\end{tabular}

